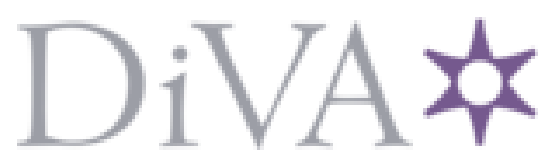

http://www.diva-portal.org

This is the published version of a paper presented at Multimedia \& Expo Workshops (ICMEW), 2015 IEEE International Conference on.

Citation for the original published paper:

Hu, Y., Peng, C., Bai, G. (2015)

Sharing Health Data Through Hybrid Cloud For Self-Management.

In:

http://dx.doi.org/10.1109/ICMEW.2015.7169752

N.B. When citing this work, cite the original published paper.

Permanent link to this version:

http://urn.kb.se/resolve?urn=urn:nbn:se:bth-11385 


\title{
SHARING HEALTH DATA THROUGH HYBRID CLOUD FOR SELF-MANAGEMENT
}

\author{
Yan Hu, Cong Peng, Guohua Bai \\ Department of Creative Technologies, Blekinge Institute of Technology, 37179, Karlskrona, Sweden \\ yan.hu@bth.se, chpd12@student.bth.se, guohua.bai@bth.se
}

\begin{abstract}
Nowadays, patient self-management is encouraged in homebased healthcare, especially for chronic disease care. Sharing health information could improve the quality of patient selfmanagement. In this paper, we introduce cloud computing as a potential technology to provide a more sustainable longterm solution compared with other technologies. A hybrid cloud is identified as a suitable way to enable patients to share health information for promoting the treatment of chronic diseases. And then a prototype on the case of type 2 diabetes is implemented to prove the feasibility of the proposed solution.
\end{abstract}

Index Terms - Chronic disease, information sharing, self-management, cloud computing, hybrid cloud

\section{INTRODUCTION}

Chronic diseases have become a main challenge to health institutions around the world. As the incidence and prevalence of chronic diseases continue to increase, traditional hospital-based healthcare is less able to meet the needs of every patient [1]. It is very inconvenient for a chronic diseases patient to be monitored frequently, especially for the aged people with more than one choric diseases. Due to the continuously increasing of chronic diseases patients, the spending on medical care is also growing rapidly[2]. Usually, it is not necessary to have urgent medical diagnosis and treatment for chronic diseases treatment. Therefore it is more appropriate to adopt effective approach to trace and control the patients' conditions via the healthcare services such as physiological signals monitoring and recording at the home environment [3].

Thanks to the development of information and communication technology (ICT), the feasibility of homebased healthcare has been highly raised. Many physiological signals can be measured by people at their living environments in daily lives [3]. If various healthcare parameters could be shared to the care providers and other people related to the home-based healthcare by the care recipients themselves at home, it will save a lot of time and resources, as well as increase the quality of chronic diseases care.

However, the process of this long-term monitoring will generate massive samples of patient self-recording health data. Consider the huge number of patients, there is a big volume of data to be mined. Most of patients do not have much knowledge on healthcare data. They may not have the ability to catch "signals" from their daily healthcare. So if they share their health data to third party for analysis, they can receive some useful feedback or some suggestions. It will help them to have a clearer understanding of their state of health. In case the patients volunteer to share their dairy health data to other healthcare research institutions or service providers, it will benefit the research of the chronic diseases, and give improved and appropriate treatments to the patients as well.

This paper begins with the description of home-based healthcare for chronic diseases. According to the comparison of different cloud deployment models in Section 3, hybrid cloud is evaluated as the most suitable model in healthcare. In Section 4, we design a prototype for healthcare recipients' self-management based on hybrid cloud deployment model. Section 5 addresses the conclusion and future work of our research.

\section{HOME-BASED CARE FOR CHRONIC DISEASES}

Chronic diseases influence patients in long last duration. Usually, lifestyle, diets and metabolism are the physical actives that cause most common chronic diseases. Treating chronic diseases depends heavily on the patients' daily behaviors. Therefore, most conditions of chronic diseases may be alleviated by changing daily behaviors, such as discontinuing the habits of smoking and drinking, implementing and regulating a healthy diet, or increasing physical exercise [4].

Home-based healthcare could enable the care recipients to live assisted home. Healthcare providers could monitor the patients based on their shared daily health data, and provide some clinical suggestions, as well as giving feedback through reports of medical examinations that the patients have undergone. In addition, for home-based healthcare, more people are encouraged to assist with the care, such as family 
members and other patients with the same symptoms. This patient-centered home-based healthcare will encourage healthcare recipients to be more active in their healthcare, involving some self-management activities and sharing the valuable health information to their care providers or other research institutions [5].

ICT evolution has led to widespread use of wireless personal devices like smartphones, personal computers and other selfmonitoring devices. This can provide a solution to help with home-based healthcare. There are already some commercial or research-based platforms for healthcare self-management such as HealthVault [6] and Apple Health [7].

\subsection{Type 2 Diabetes}

Diabetes is a disease that patients need to be monitored continuously for a number of parameters, such as blood sugar level, blood pressure and so on. Simultaneously, the effect of environmental factors such as nutrition, stress and exercise should also be taken into account when managing this disease [8]. It would be beneficial and efficient if these various aspects of parameters could be recorded by the patients themselves at homes. According to the report by WHO, almost 1 in 10 adult has diabetes in the world, measured by elevated fasting blood glucose $(>=126 \mathrm{mg} / \mathrm{dl})$. People with diabetes have increased risk of heart disease and stroke, and are 10 times more likely to need a lower limb amputation than people who do not have diabetes. It is also one of the leading causes of renal failure, visual impairment and blindness.

According to the information gathered by C. Nordqvist [9], lacking of enough proper functioned insulin production, the cells in the body have improper respondence to insulin, or both, are the reasons cause diabetes. There are three types of diabetes: type 1 diabetes, type 2 diabetes and gestational diabetes. Over $90 \%$ of all cases of diabetes worldwide are type 2 diabetes. Most type 2 diabetes patients will have the condition of prediabetes first. The blood glucose level of a person had prediabetes is higher than normal, but not high enough for a diabetes diagnosis. As one kind of chronic disease, type 2 diabetes will get worse gradually. In addition, obesity causes the body to release chemicals which will destabilize the bodies cardiovascular and metabolic systems, this will lead to a much higher risk of developing to type 2 diabetes [9].

People could control their symptoms of type 2 diabetes by keeping a healthy diet, losing weight, doing a plenty of exercises, and monitoring blood glucose level [9]. Therefore, it is possible that patients can self-manage at home by daily recording blood glucose level parameter, diet, insulin intake, physical exercise, feet checking, psychological status (such as mood and pressure) and living habits (such as smoking and alcohol abuse), and then sharing to healthcare providers. This study will start with the self-management of type 2 diabetes to prove the feasibility and practicality of the notion, and then try to apply the pattern to other chronic diseases.

\section{CLOUD COMPUTING IN EHEALTH}

Several benefits will be brought by adopting cloud computing in the current healthcare systems, such as health information management system, laboratory information system, pharmacy information system and so on [10].

Cloud computing "can provide distributed, rapidly provisioned and configurable computing resources (such as servers, storage, applications, networks and other services), which are on-demand, rapid elastic and measured, to whom have network connections" [11]. The obvious advantages like high scalability, availability and security of cloud services, make it a trend of applying cloud computing in eHealth area since last few years[12]. Since the patient daily health data is in a huge amount, cloud storage service seems to be a preferable way to sharing of these data among hospitals and healthcare organizations or third party research institutions. The big healthcare data in the cloud can be processed by remote services, such as different IT systems in hospital and other healthcare organizations, as well as some third party online care services. These features give more reasons to adopt cloud computing in sharing health information.

\subsection{Comparison of different cloud deployment models}

There are four cloud deployment models: public, private, community and hybrid clouds. Each model has its own advantages and drawbacks. Since in the healthcare domain, deploying a community cloud will bring obvious problems, due to it involves multiple different organizations. Third party organizations and hospitals have different properties and duties by nature. They have their own different interests beyond a common shared mission and compliance. Accordingly, allocation of accountability and responsibility will be confusing when problems occur for either administration or management. Therefore, the community cloud deployment model will not be considered in our comparison. For the other three cloud deployment models, the comparison will be conducted through analyzing and comparing the following five factors: security and privacy, scalability and capability, customization, costs of setup and maintenance, and legal issues.

\subsubsection{Security and Privacy}

Security and privacy of healthcare data is one of the key factors in building the trust of patients. A public cloud is hosted by a third party enterprise, the health data will be stored in the servers, which are not fully trustable and are out of control of both the healthcare providers and the recipients. This will increase the potential for sensitive information leakage. The third party storage servers are often the targets of malicious attacks, which will be even worse due to the high value of the sensitive health information. Due to these facts, patients are probably not willing to let their entire health data 
be stored in public cloud servers. A private cloud is usually hosted by the organization in-house. The health data will be stored in the servers are operated by healthcare service providers. Although the disaster tolerance is not good as a public cloud, the threat of outside malicious attack will be far less. In addition, it is more flexible and reliable to implement fine-grained access control mechanisms to protect the privacy of health data. A hybrid hosts sensitive data and workflow in its private cloud part, and can provide the same security and privacy guarantee as a private cloud.

\subsubsection{Scalability and Capability}

Another important reason for adopting cloud computing in health information sharing is the scalability and powerful capabilities. Because of the benefits of distributed massive computer clusters, ubiquity and virtualization, a public cloud can provide high performance services with low requirements on user-end computer infrastructure. Compared with the economic reasons, the capability of the development platform is an important factor to adopt a public cloud [13]. Third party eHealth service providers can develop various services, such as historical health analysis, health data mining and Clinical Decision Support System [13], on a public cloud platform. Theoretically, a private cloud can provide the same features as a public cloud. However, due to the private cloud's own characteristics of smaller scale and limited access, it is unrealistic to make a private cloud function as a platform which needs to host various third party services [14]. This will cut down the potential value of massive health data. If a hybrid cloud hosts shared health information and insensitive services in its public cloud part, it can provide approximately the same scalability and capabilities as a public cloud.

\subsubsection{Customization}

Different legacy systems are used by different healthcare providers, and the deployed cloud model needs to be integrated into these systems without impacting the previous workflow. Although public cloud providers deliver a set of various services, it is hardly to satisfy the special requirements from different healthcare providers. A private cloud provides the capability to customize services in accordance with particular demands. The hospital can modify the cloud system to be coordinated with other legacy systems, and can even move the legacy systems to a private cloud. A hybrid cloud has the ability to keep the capability of customization, while at the same time migrating appropriate services to the public cloud part. This makes a hybrid cloud even more flexible than a private cloud.

\subsubsection{Cost}

A public cloud offers a pay-per-usage charging model. Different parties only need to pay for the services they have used. The initial setup costs of hardware, software and bandwidth are covered by public cloud providers. Moreover, the lengthy time consumption and costly long-term maintenance, as well as the update of software and hardware, are no longer bottomless values. The costs of expansion, updates and long-term maintenance will be a burden to an organization which uses a private cloud. The investment cost of adopting a hybrid cloud is approximate to that of a private cloud [15]. However, the migration of insensitive data and services to the public cloud part will reduce some of the burden as compared to a pure private cloud.

\subsubsection{Legal}

At present, a majority of big public cloud providers are from the US, so most data centers are located in the US. Because of the specialty of health data, it may cause some potential legal risks for other countries to deploy a public cloud in healthcare [16]. Different countries have different laws and regulations on managing patient data, and some nations do not allow sensitive health data to be transferred cross-border. Although big public cloud providers such as Amazon may offer options to allow customers to choose the regions for the storage of data, the choices of region are still limited. A private cloud will be hosted by healthcare organizations, so there will be no concern about the violation of laws or other regulations. As for a hybrid cloud, the sensitive data can be kept in the private cloud part while utilizing the public cloud part to process insensitive data.

Based on the analysis and comparison of the five key factors, it can be summarized that a public cloud is less appropriate than the other two clouds in security and privacy, customization and legal issues. A private cloud is less appropriate than the other two clouds in scalability and cost. By combing a private cloud with a public cloud, a hybrid cloud seems to be the most appropriate cloud deployment model for the objective of sharing health data. We use a quantitative score evaluation method [17] to compare these three cloud models, as shown in the radar chart below.

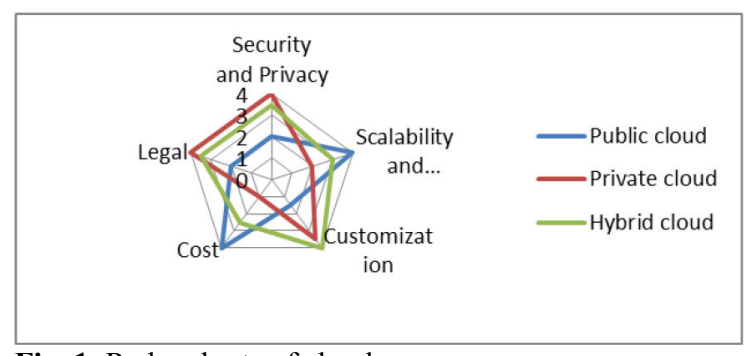

Fig. 1. Radar charts of clouds.

\section{PROTOTYPE DESIGN}

A prototype is defined as an early model or demonstration of a system which is built to test the feasibility of a concept, solution or process [18]. It has been widely used in system development and research. In this section, the whole structure of the hybrid cloud platform will be designed and depicted 
for a clear demonstration of the expected purpose. Then an implemented vertical prototype will be presented, which is though incomplete, with the key functions of recording health information, sharing to private cloud and optional sharing to public cloud.

\subsection{Conceptual Scenario of Health Information Sharing}

The Fig. 2 below describe a conceptual scenario that how the self-management healthcare data will be shared to a hospital or other care providers (private cloud) and third party healthcare service or research institution (public cloud) in order to receive home-based healthcare services, improve the treatments as well as contribute to researches.

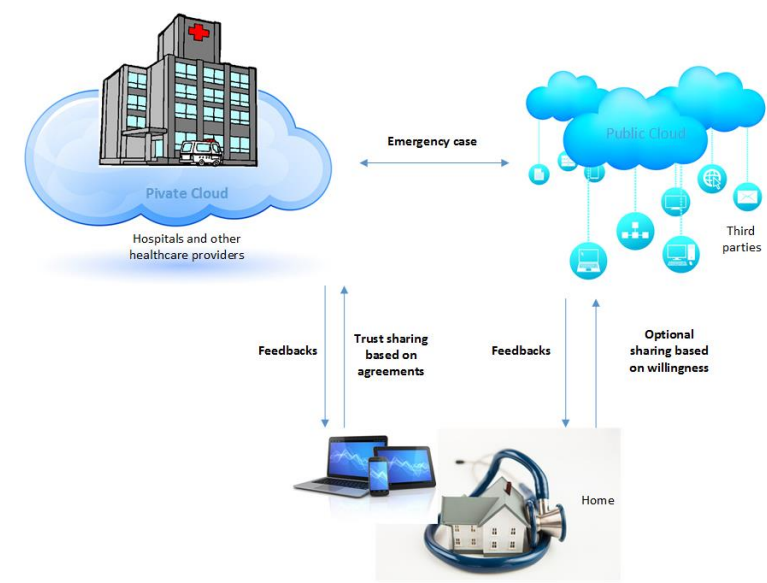

Fig. 2. Conceptual Scenario of Sharing Health Information.

\subsubsection{Share to Hospital or other care providers}

Based on the eHealth policies in EU and Sweden [19], we suggest that the healthcare recipients should have some trust agreements with their providers before they sharing daily healthcare data to the hospitals and other healthcare organizations' private cloud. Because some care providers like doctors need a complete record including personal information from patients. In most cases, nurses or other medical personals are involved this home-based case, they just want the patients' data related to their services. The agreements is including all these issues. The healthcare providers will also guarantee a fine-grained data access control mechanism and security protection techniques to keep the privacy and security of the shared information in the private cloud. The shared health information could be integrated into electronic records in hospitals and healthcare centers.

\subsubsection{Share to Third Parties}

As known to all, privacy and security are patients' greatest concerns [20]. Besides these, when healthcare recipients considering to share their personal health information to the third parties of public cloud which were not fully trusted, the most important thing is the control rights of data. Personal information is inappropriate to be shared in the public cloud, so a tracking ID is the identification. To make the shared health data meet the requirements of specific purposes is a key feature because it is not necessary to share all the homebased healthcare data to the third parties.

\subsubsection{Share among Healthcare Providers and Third Parties}

When the health information needed to be shared to another organization, it is important to inform the care recipients with reasons. Then the action of sharing should be authorized by the patient before the transferring of health data. In this scenario, a patient's health record could be shared between third party organizations and healthcare providers in some emergency cases. Every time when a health record is shared in an emergency case, it needs to be recorded for verification.

\subsection{System Design \\ 4.3.1 Health Record Structure}

Table 1 shows each element of the patient-recorded health record and which cloud deployment they are mainly stored. The elements are mainly referenced from the CCD template[21] and PatientsLikeMe [22]user charts.

Table 1. Health Record Structure

\begin{tabular}{|c|c|c|}
\hline Record Element & Content & $\begin{array}{c}\text { Cloud } \\
\text { Model }\end{array}$ \\
\hline $\begin{array}{c}\text { Record } \\
\text { Identification }\end{array}$ & $\begin{array}{c}\text { Personal information, } \\
\text { tracking ID }\end{array}$ & Private \\
\hline $\begin{array}{c}\text { Personal health } \\
\text { status }\end{array}$ & $\begin{array}{c}\text { Diagnosed diseases, other } \\
\text { health related information }\end{array}$ & Private \\
\hline $\begin{array}{c}\text { Physiologic } \\
\text { measurements }\end{array}$ & $\begin{array}{c}\text { Blood pressure, blood } \\
\text { glucose, heart Rate, etc. }\end{array}$ & Private, \\
\hline $\begin{array}{c}\text { Non- } \\
\text { physiologic } \\
\text { records }\end{array}$ & $\begin{array}{c}\text { Mood, pain, fatigue, } \\
\text { insomnia, blurry vision or } \\
\text { other related symptoms }\end{array}$ & Private, \\
\hline $\begin{array}{c}\text { General health } \\
\text { measurements }\end{array}$ & $\begin{array}{c}\text { Weight, height, } \\
\text { cholesterol, etc. }\end{array}$ & Public \\
\hline Medication \\
Dosage & $\begin{array}{c}\text { Amounts of injected } \\
\text { insulin per day, other } \\
\text { medicines information }\end{array}$ & Private \\
\hline $\begin{array}{c}\text { Life style } \\
\text { records }\end{array}$ & $\begin{array}{c}\text { Food records and } \\
\text { physical activities }\end{array}$ & Public \\
\hline $\begin{array}{c}\text { Family health } \\
\text { history }\end{array}$ & $\begin{array}{c}\text { Diseases and health } \\
\text { situations occurred to } \\
\text { immediate families }\end{array}$ & Private \\
\hline $\begin{array}{c}\text { Patient notes } \\
\text { information above }\end{array}$ & Public \\
\hline
\end{tabular}


When sharing to a public cloud, the Personal information (includes name, address, etc.) of the Record identification will be removed for personal's privacy protection. The Tracking ID is a randomly-generated global-unique ID. This will be used to track the patients in the emergency case. Before sharing to the public cloud, all other elements in the record could be choose to share or not based on the willingness of patients. The format of this health record is inherited from HL7-CCD and contained in an XML file.

\subsection{Prototype Simulation}

On the preliminary stage of the whole hybrid cloud platform, it is necessary to establish the connections among all the roles involved for exploration. Therefore an initial prototype is implemented, which is composed of a simple patient client, an OpenStack system with all components hosted in virtual machine, and Amazon Web Services. The patient client has been implemented with the functions of recording health information, sharing to private cloud and optional sharing to public cloud. The structure of health data inherits from HL7CCD. The prototype is programmed by Java, with AWS SDK, open source libraries dom4j [23] for XML parsing and Joss [24] for OpenStack Swift API.

\subsubsection{Health Data Recording and Sharing}

Since this version of prototype is used to test the key functions of the incomplete working system, the interface of patient client is kept in simple. One of the interfaces is shown in Fig. 3. This interface classifies some health record elements, which are defined in section 4.3.1. After inputting health data, patients can save the record in local as an CCD similar XML file, upload the record to private cloud, or press the "Sharing Options" button to share to public cloud, which will be performed in another interface.

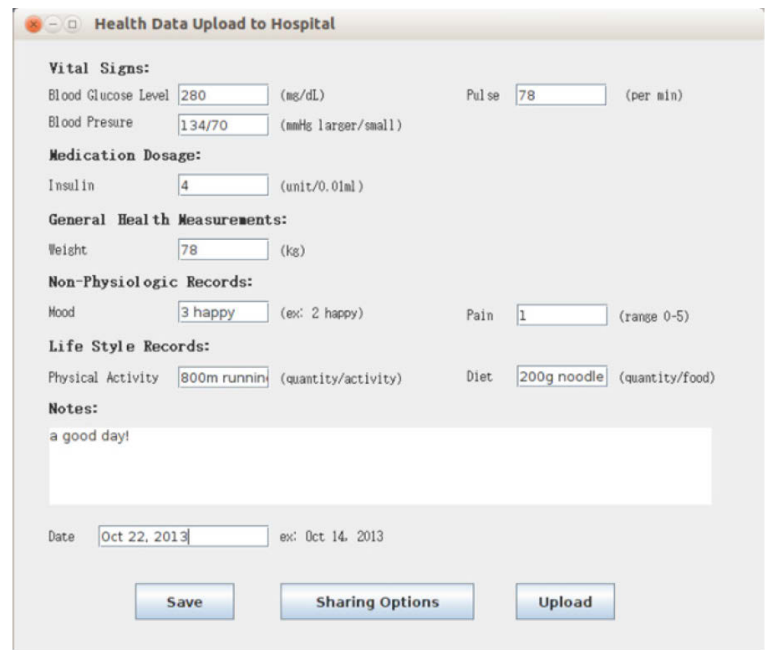

Fig. 3. Interface of Recording and Sharing to Private Cloud
The "Sharing Options" is shown in Fig. 4. In this interface, patients can choose which health record elements to share based on their willingness. Patients can also choose where they want to share or what service they want to receive, such as diabetes research lab or clinical decision support system.

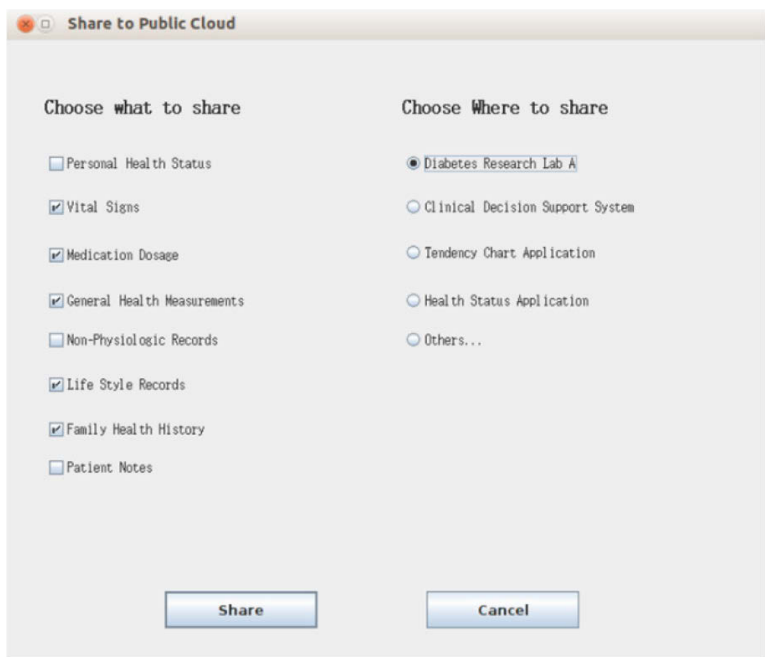

Fig. 4. Interface of Choosing and Sharing to Public Cloud

\section{CONCLUSION AND FUTURE WORK}

In home-based healthcare, self-management is becoming an important approach for chronic patients to deal with their diseases by continuously monitoring and recording health vital signs and other related data. To share the patientrecorded health information is the key process to promote the treatments of chronic diseases, either sharing to healthcare providers or other healthcare related organizations. For addressing these problems, we implemented a vertical prototype of the conceptual hybrid cloud solution. As a result, the prototype, which is developed with OpenStack and AWS, successfully worked with the functions of recording home-based health information, sharing to the private cloud and optional sharing to the public cloud. Through researching particularly with type 2 diabetes, we believe the proposed solution is also feasible to manage chronic diseases just with slight modification, since the method of segmenting valuable and suitable health data is similar.

To integrate with automatic health devices and personal mobile devices is a vital task of home-based chronic disease self-management in the future. The automatic way of health data collection is not only to facilitate patients to record massive data, but also to reduce errors due to lacking of medical knowledge. This kind of cloud based solution is a driving force for interoperability and no patient data locked in one product. And all the data will have an entire backup in private cloud and in patient client devices. It gives patients their own controls over their data. However, to make the data 
interoperability at an international level, it requires worldwide organizations to coordinate various applications from local level. For achieving an interoperable solution, common medical coding system standards and health record standards such as CDA or CCD are should be implemented in the solution for electronic health information exchange.

\section{REFERENCES}

[1] "What is Chronic Disease? | Center for Managing Chronic Disease - Putting People at the Center of Solutions," What is Chronic Disease? | Center for Managing Chronic Disease - Putting People at the Center of Solutions. [Online]. Available: http://cmcd.sph.umich.edu/what-is-chronicdisease.html. [Accessed: 28-May-2014].

[2] P. J. Cunningham, "The Share Of People With High Medical Costs Increased Prior To Implementation Of The Affordable Care Act," Health Aff. (Millwood), vol. 34, no. 1, pp. 117-124, Jan. 2015.

[3] Z. Pang, L. Zheng, J. Tian, S. Kao-Walter, E. Dubrova, and Q. Chen, "Design of a terminal solution for integration of in-home health care devices and services towards the Internet-of-Things," Enterp. Inf. Syst., vol. 9, no. 1, pp. 86-116, Jan. 2015.

[4] G. Danaei, E. L. Ding, D. Mozaffarian, B. Taylor, J. Rehm, C. J. Murray, and M. Ezzati, "The preventable causes of death in the United States: comparative risk assessment of dietary, lifestyle, and metabolic risk factors," PLoS Med., vol. 6, no. 4, p. e1000058, 2009.

[5] C. R. Weir, N. Staggers, B. Gibson, K. Doing-Harris, R. Barrus, and R. Dunlea, "A qualitative evaluation of the crucial attributes of contextual Information necessary in EHR design to support patient-centered medical home care," BMC Med. Inform. Decis. Mak., vol. 15, no. 1, p. 30, Apr. 2015.

[6] "HealthVault," HealthVault. [Online]. Available: https://www.healthvault.com/se/en. [Accessed: 02-Jul2014].

[7] “Apple - iOS 8 - Health," Apple. [Online]. Available: https://www.apple.com/ios/whats-new/health/. [Accessed: 13-May-2015].

[8] S. Deo, D. Deobagkar, and D. D. Deobagkar, "Design and development of a web-based application for diabetes patient data management," Inform. Prim. Care, vol. 13, no. 1, pp. 35-41, Feb. 2005.

[9] "What is Diabetes? What causes Diabetes?" [Online]. Available:

http://www.medicalnewstoday.com/info/diabetes/. [Accessed: 06-Feb-2015].

[10] A. Bahga and V. K. Madisetti, "A Cloud-based Approach for Interoperable Electronic Health Records (EHRs)," IEEE J. Biomed. Health Inform., vol. 17, no. 5, pp. 894-906, Sep. 2013.
[11] P. Mell and T. Grance, "The NIST definition of cloud computing," Natl. Inst. Stand. Technol., vol. 53, no. 6, p. 50, 2009.

[12] Y. Hu and G. Bai, "A Systematic Literature Review of Cloud Computing in eHealth," Health Inform.- Int. J. HIIJ, vol. 3, no. 4, pp. 11-20, Nov. 2014.

[13] M. Hussain, A. M. Khattak, W. A. Khan, I. Fatima, M. B. Amin, Z. Pervez, R. Batool, M. A. Saleem, M. Afzal, M. Faheem, M. H. Saddiqi, S. Y. Lee, and K. Latif, "Cloud-based Smart CDSS for chronic diseases," Health Technol., vol. 3, no. 2, pp. 153-175, Jun. 2013.

[14] "Compare the Cloud - ComparetheCloud.net," Compare the Cloud - ComparetheCloud.net. [Online]. Available: http://www.comparethecloud.net/. [Accessed: 17-Sep2014].

[15] O. Mazhelis and P. Tyrväinen, "Economic aspects of hybrid cloud infrastructure: User organization perspective," Inf. Syst. Front., vol. 14, no. 4, pp. 845869, Sep. 2012.

[16] Z. Mahmood, "Data Location and Security Issues in Cloud Computing," in 2011 International Conference on Emerging Intelligent Data and Web Technologies (EIDWT), 2011, pp. 49-54.

[17] "Therapeutic intervention scoring system: a method for quanti...: Critical Care Medicine," Therapeutic intervention scoring system: a method for quanti...: Critical Care Medicine. [Online]. Available: http://journals.lww.com/ccmjournal/Fulltext/1974/0300 0/Therapeutic_intervention_scoring_system_a_metho d.1.aspx. [Accessed: 21-Oct-2014].

[18] "What is Prototype model- advantages, disadvantages and when to use it?," What is Prototype modeladvantages, disadvantages and when to use it?. [Online]. Available: http://istqbexamcertification.com/what-is-prototypemodel-advantages-disadvantages-and-when-to-use-it/. [Accessed: 22-Sep-2014].

[19] W. L. Currie and J. J. M. Seddon, "A cross-national analysis of eHealth in the European Union: Some policy and research directions," Inf. Manage., vol. 51, no. 6, pp. 783-797, Sep. 2014.

[20] C. Wang, X. Liu, and W. Li, "Implementing a Personal Health Record Cloud Platform Using Ciphertext-Policy Attribute-Based Encryption," in 2012 4th International Conference on Intelligent Networking and Collaborative Systems (INCoS), 2012, pp. 8-14.

[21] "Continuity of Care Document (CCD) - Templates." [Online]. Available: http://art-decor.org/artdecor/decor-templates--ccd1-. [Accessed: 06-Feb2015].

[22] "PatientsLikeMe." [Online]. Available: www.patientslikeme.com/.

[23] "dom4j." [Online]. Available: http://www.dom4j.org/.

[24] "javaswift/joss . GitHub." [Online]. Available: https://github.com/javaswift/joss. [Accessed: 11-Feb2015]. 